\title{
Benefícios da Massagem Modeladora na Lipodistrofia Localizada
}

\author{
Aiana Tátima Oliveira Mota Machado ; Ana Paula Silva Nogueira ${ }^{2}$ : Luciana Trindade de Santana Leão \\ Beluzia Almeida Santos ${ }^{4}$; Livia Mara Gomes Pinheiro ${ }^{5}$; Sirlândia Santos Oliveira ${ }^{6}$
}

Resumo: Resumo: A lipodistrofia localizada consiste no acúmulo de gorduras em certas regiões do corpo. A massagem modeladora é um dos procedimentos existentes para tratar essa disfunção. É uma técnica que possui o objetivo de promover a mobilização da gordura, aumento da circulação vascular periférica e eliminação de toxinas. Seus resultados são potencializados quando associada à ativos como castanha da índia, centella asiática, extrato de cavalinha e cafeína. Esta pesquisa trata-se de um projeto de intervenção com abordagem quantitativa. Foram selecionadas duas voluntárias para colaborar com a presente pesquisa, que foram submetidas a oito sessões, duas vezes por semana, de um tratamento com creme redutor a base de castanha da índia, centella asiática, extrato de cavalinha e cafeína, e manobras de massagem modeladora. Os resultados demonstraram redução na circunferência abdominal e melhora do aspecto da pele. Portanto, concluiu-se que a massagem modeladora é uma técnica eficaz no tratamento da lipodistrofia localizada.

Palavras-chave: Gordura Localizada. Cosmetologia. Massagem Modeladora. Lipodistrofia Abdominal. Lipólise.

\section{Benefits of Modeling Lipodystrophy Massage Localized}

\begin{abstract}
Localized lipodystrophy consists of the accumulation of fats in certain regions of the body. Modeling massage is one of the existing procedures to treat this dysfunction. It is a technique that aims to promote the mobilization of fat, increase peripheral vascular circulation and elimination of toxins. Its results are potentiated when associated with assets such as india nut, asian centella, horsetail extract and caffeine. This research is an intervention project with a quantitative approach. Two volunteers were selected to collaborate with the present research, who were submitted to eight sessions twice a week of a treatment with reducing cream based on indian chestnut, asian centella, horsetail extract and caffeine, and maneuvers of modeling massage . The results demonstrated a reduction in the waist circumference and improvement of the appearance of the skin. Therefore, it has been concluded that modeling massage is an effective technique in the treatment of localized lipodystrophy.
\end{abstract}

Keywords: Localized Fat. Cosmetology. Modeling Massage. Abdominal Lipodystrophy. Lipolysis.

\section{Introdução}

A busca pelo corpo perfeito vem aumentando ao decorrer dos anos. Tanto o público feminino quanto o masculino têm recorrido a diversos tratamentos. Segundo Tacani et al. (2010), atualmente existem padrões de beleza determinados pela sociedade e a partir destes tem crescido a busca pelos cuidados estéticos.

\footnotetext{
${ }^{1}$ Graduanda em Estética e Cosmética pela Faculdade Independente do Nordeste - FAINOR. Autor correspondente: E-mail: aianafafa@hotmail.com. Fone - ( 77) 99101-2653, Vitória da Conquista, Bahia, Brasil.

${ }^{2}$ Fisioterapeuta dermato-funcional. Especialista em unidade de terapia intensiva - UTI. Vitória da Conquista, Bahia, Brasil.

${ }^{3}$ Esteticista. Especialista em metodologia do ensino superior. Vitória da Conquista, Bahia, Brasil.

${ }^{4}$ Enfermeira. Especialista em docência do ensino superior e EAD. Vitória da Conquista, Bahia, Brasil.

${ }^{5}$ Enfermeira. Especialista em urgência e emergência. Especialista em saúde pública com ênfase em PFS. Especialista em saúde mental.

Especialista em ensino superior na área de saúde. Vitória da Conquista, Bahia, Brasil.

${ }^{6}$ Fisioterapeuta dermato-funcional. Vitória da Conquista, Bahia, Brasil.
} 
De forma geral, é notório como as pessoas sempre estão insatisfeitas com algo em sua estética corporal. Umas são mais exigentes, outras menos, mas a maioria destas deseja modificar algo em seu corpo, sendo a principal queixa o excesso de gordura, ou seja, a lipodistrofia localizada, conhecida também como gordura localizada (TOGNI, 2006).

Guirro e Guirro (2004) explicam que o tecido adiposo é formado por tecido conjuntivo e células comumente chamadas de adipócitos. São encontrados isolados ou em conjunto e até mesmo aglomerados em amplas regiões do corpo. $\mathrm{O}$ aumento desordenado do tecido conjuntivo adiposo subcutâneo é chamado de lipodistrofia localizada.

A distribuição de gordura não é uniforme em todas as regiões do corpo. Nos indivíduos normais, algumas regiões nunca acumulam gordura, como a pálpebra, a cicatriz umbilical, o pênis e as dobras articulares. Em outras regiões, pelo contrário, há maior acúmulo de tecido adiposo: a porção proximal dos membros, a parede abdominal, especialmente as porções laterais. No sexo feminino há em média 8 a $10 \%$ mais gordura corporal do que no masculino. Nas mulheres, a gordura essencial representa entre 9 a $12 \%$ e nos homens 3\%, isso ocorre devido a diferenciação hormonal de cada sexo (SANDOVAL, 2005).

A lipodistrofia localizada é classificada pelo espaço em que está posicionada, podendo ser: andróide ou central, na qual a gordura se encontra no abdômen e é mais comum no sexo masculino; ginóide ou periférica, seu acúmulo está nas coxas e quadril, comumente encontrado em mulheres; e mista, quando há associação da andróide e ginóide (MELLO et al., 2010).

Seu aparecimento está diretamente relacionado com o sedentarismo, stress, antecedentes familiares, tabagismo, alterações hormonais, síndrome pré-menstrual, anticoncepcionais, elevação do estrógeno, androsterona, insulina dentre outras (RIBEIRO, 2006). Guirro e Guirro (2004) acrescentam que o acúmulo de gorduras nas células está associado à quantidade insuficiente de enzimas que estão sendo produzidas para a queima dessas gorduras. Assim, os tratamentos estéticos para a gordura localizada visam aumentar a produção destas enzimas para que ocorra maior combustão das gorduras em excesso.

Dentre os procedimentos estéticos mais utilizados nos dias atuais, está a massagem modeladora. Essa massagem é baseada no uso de diversas técnicas manuais com objetivo de promover a mobilização da gordura, aumento da circulação vascular periférica e auxiliar na eliminação de toxinas (BORGES, 2006). 
De acordo com Borges (2006), a massagem modeladora melhora a circulação, facilita o retorno venoso, melhora a aparência corporal e o bem estar. Produz melhores resultados quando é executada corretamente e em conjunto com ativos cosméticos. Segundo Guirro e Guirro (2004), os ativos farmacológicos utilizados na terapêutica da lipodistrofia localizada atuam no tecido conjuntivo ou na microcirculação, podendo ser utilizados por vias tópica, sistêmica ou transdérmica.

Borges (2006) comenta que a cosmetologia utiliza ativos de grande permeação, capazes de estimular o aumento da circulação sanguínea e linfática, podendo também promover a desagregação das fibroses de gordura acelerando a hidrólise do excesso de gordura na formação de ácidos graxos. Guirro e Guirro (2004) afirmam que os cosméticos podem aumentar a produtividade das enzimas que participam do processo de redução do acúmulo de gordura. Os principais ativos empregados são castanha da índia, centella asiática, extrato de cavalinha e cafeína.

A lipodistrofia localizada é a principal alteração estética que se percebe nos dias atuais, podendo existir até em indivíduos magros, e muitas vezes está associada a problemas psicológicos e sociais, pois aos olhos críticos, está fora do padrão de beleza imposto pela sociedade, e estes motivos levam à busca por tratamentos estéticos capazes de melhorar essa disfunção (NEVES; OLIVEIRA, 2008).

A massagem modeladora promove benefícios como a oxigenação do tecido, quebra de cadeias de gordura e a melhora do tônus muscular, favorecendo assim não só a estética corporal, mas também agindo no psicológico do cliente diminuindo o estresse e proporcionando ao mesmo bem-estar com saúde.

\section{Métodos}

Trata-se de um projeto de intervenção com abordagem quantitativa. Traduz-se por tudo aquilo que pode ser quantificável, ou seja, exprime em números as opiniões e informações para então obter a análise dos dados e, posteriormente, chegar a uma conclusão.

A coleta de dados foi realizada em um núcleo de Estética e Cosmética de uma faculdade privada de Vitória da Conquista/BA. Foram selecionados 2 (dois) voluntários do sexo feminino, 
com idade entre 20 e 40 anos para colaborar com a presente pesquisa, seguindo assim aos seguintes critérios de inclusão: mulheres que apresentavam gordura localizada na região abdominal, verificada através da palpação e inspeção visual. E como critérios de exclusão: mulheres que ao decorrer da pesquisa realizassem outros procedimentos estéticos, mulheres que não apresentavam excesso de adiposidade no abdômen, mulheres gestantes e que estavam amamentando.

O tratamento teve início com a assinatura do Termo de Consentimento Livre e Esclarecido (TCLE) pelas voluntárias. Como forma de avaliação as duas voluntárias foram sujeitas a um questionário (anamnese). Em seguida, foi realizada a avaliação física, visual e palpação da região abdominal. Posteriormente foi feita a higienização e esfoliação do local a ser manipulado; foi aplicado um creme redutor a base de castanha da índia, centella asiática, extrato de cavalinha e cafeína, e logo após foram realizadas manobras de massagem modeladora. Foram feitas 8 sessões, duas vezes por semana e com duração de 40 minutos cada. Os dados foram coletados no período de abril de 2017 a maio de 2017.

Os registros fotográficos foram feitos na primeira e última sessão para comprovação da evolução das pacientes com o protocolo proposto acima. Foi realizada a perimetria pré e póstratamento. A análise dos dados foi feita através da análise de conteúdo de Bardin e das fotografias.

O presente trabalho respeitou as normas e diretrizes para a realização de pesquisas envolvendo seres humanos, correspondente a Resolução do Conselho Nacional de Saúde 466/2012. O projeto foi encaminhado e aprovado pelo Comitê de ética em pesquisa da FAINOR (Parecer $n^{\circ}$ 2.015.304).

\section{Resultados}

Diante dos dados coletados, observou-se que as duas voluntárias obtiveram um resultado positivo através da massagem modeladora associada ao creme redutor, ambas apresentaram grande redução na circunferência abdominal ao final do tratamento.

Quanto à perimetria, foram realizadas três medidas (supra-umbilical, infra-umbilical e cintura). Na perimetria pré-tratamento, a voluntária A tinha $83,5 \mathrm{~cm}$ na região supra-umbilical, 
$99 \mathrm{~cm}$ na região infra-umbilical e $86 \mathrm{~cm}$ de cintura. Na perimetria pós-tratamento, apresentou $81 \mathrm{~cm}$ na região supra-umbilical, reduzindo $2,5 \mathrm{~cm} ; 93 \mathrm{~cm}$ na região infra-umbilical, reduzindo $6 \mathrm{~cm}$; e $79 \mathrm{~cm}$ de cintura, reduzindo $7 \mathrm{~cm}$. Estes resultados estão elencados nas Figuras 1 e 2.

Os resultados da voluntária B podem ser observados nas Figuras 3 e 4. Na primeira sessão do tratamento, a voluntária apresentava as seguintes medidas: $82 \mathrm{~cm}$ na região supraumbilical, 92,5 cm na região infra-umbilical e $83 \mathrm{~cm}$ de cintura. Na última sessão, houve uma redução de $3 \mathrm{~cm}$ na região supra-umbilical, $5,5 \mathrm{~cm}$ na região infra-umbilical e 4,5 cm na cintura, sendo que a voluntária ficou com $79 \mathrm{~cm}, 87 \mathrm{~cm}$ e 78,5 cm nas respectivas regiões.

Nota-se que na região infra-umbilical houve uma maior redução de medidas do que nas outras áreas do abdômen, pois segundo Gomes e Damazio (2009) esta é uma região que apresenta maior acúmulo de gordura das mulheres, que está ligado à capacidade de procriação onde este acúmulo tem como objetivo proteger o feto.

As voluntárias sentiram desconforto e dor abdominal apenas no dia seguinte após a primeira sessão. Ambas mostraram-se satisfeitas com o resultado do tratamento e relataram que antes a gordura apresentava aspecto duro e após as oito sessões, a mesma se tornou mais maleável. Além disso, descreveram uma melhora na aparência da pele e redução da gordura localizada.

É importante ressaltar que as voluntárias seguiram alguns cuidados diários imprescindíveis para potencializar os resultados do tratamento proposto no presente trabalho. As mesmas estavam praticando atividade física três vezes por semana e tendo uma alimentação saudável. 
Id on Line Revista Multidisciplinar e de Psicoloqia

Id on Line Multidisciplinary and Psycology Journal

Figura 1. Registro fotográfico da voluntária A, antes e depois do tratamento (região frontal e dorsal).

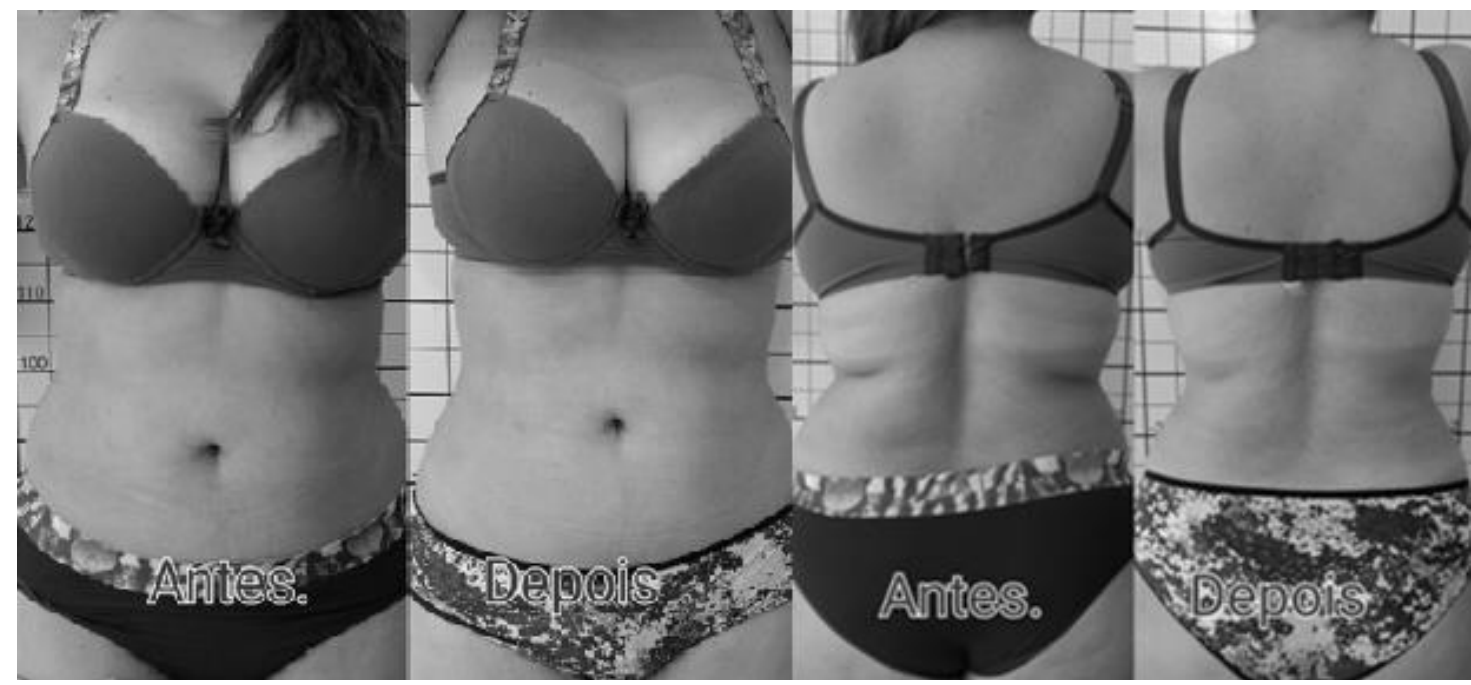

Figura 2. Registro fotográfico da voluntária A, antes e depois do tratamento (região lateral direita e esquerda).

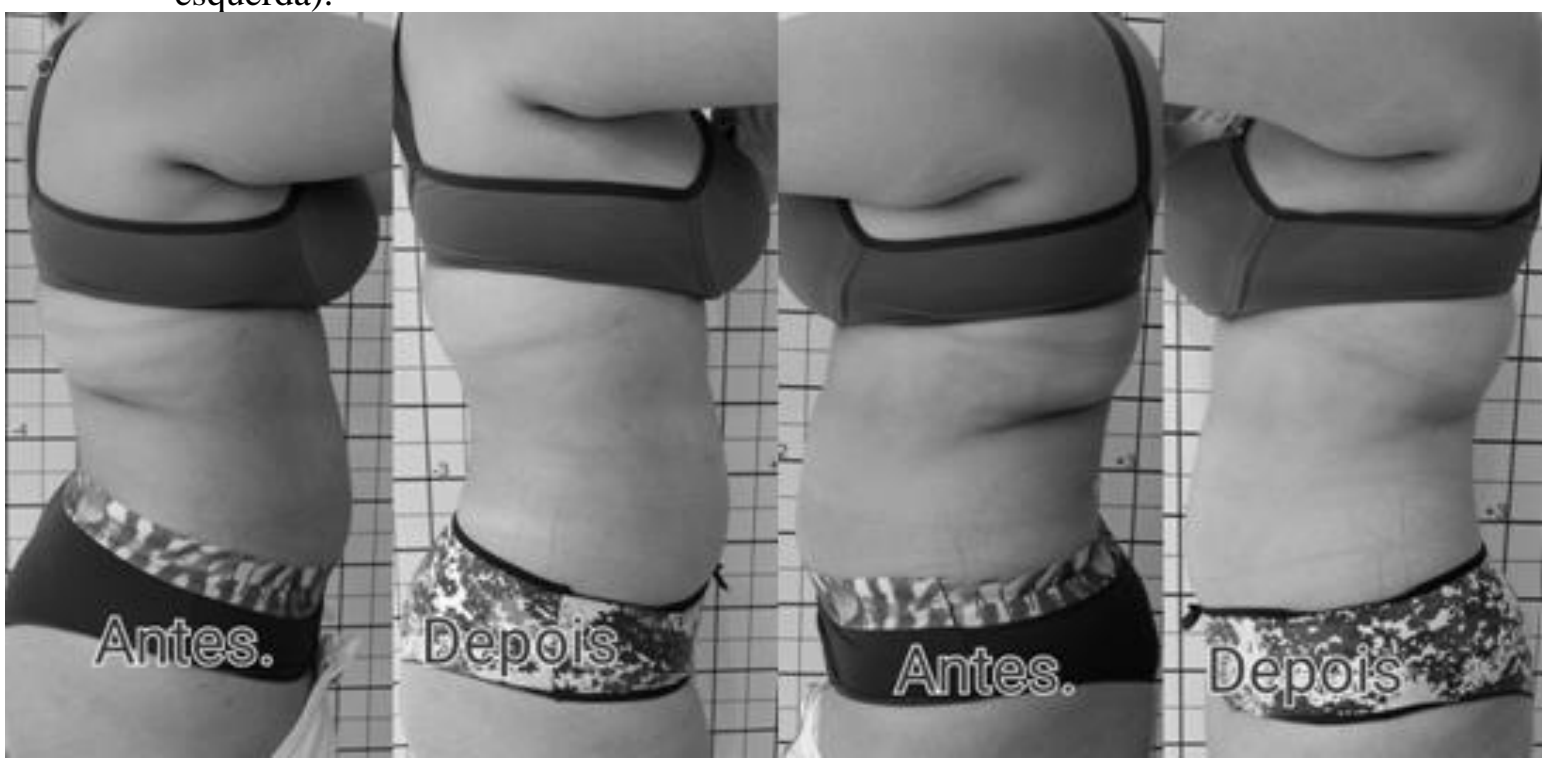


Id on Line Revista Multidisciplinar e de Psicoloqia

Id on Line Multidisciplinary and Psycology Journal

Figura 3. Registro fotográfico da voluntária $\mathrm{B}$, antes e depois do tratamento (região frontal e dorsal).

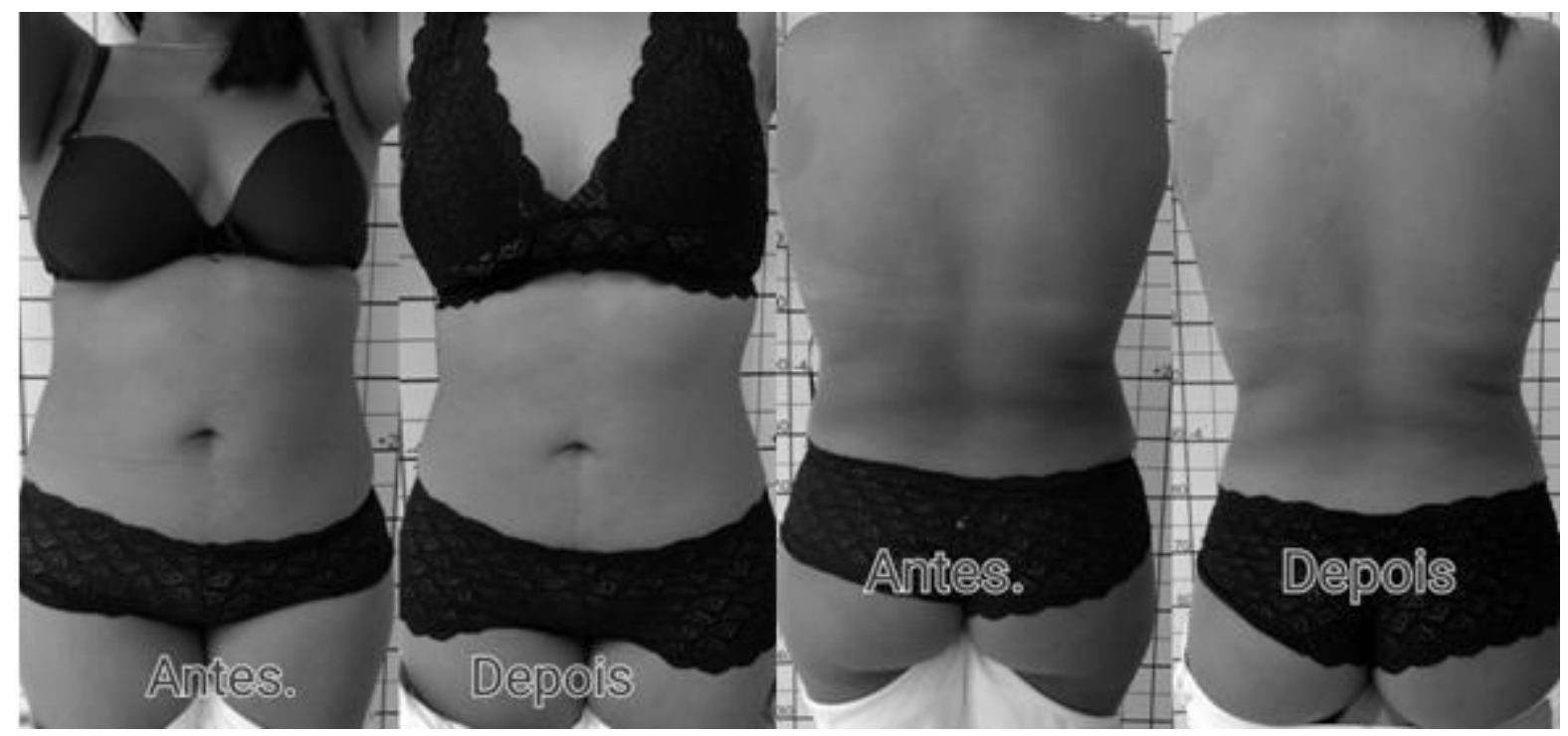

Figura 4. Registro fotográfico da voluntária B, antes e depois do tratamento (região lateral direita e esquerda).

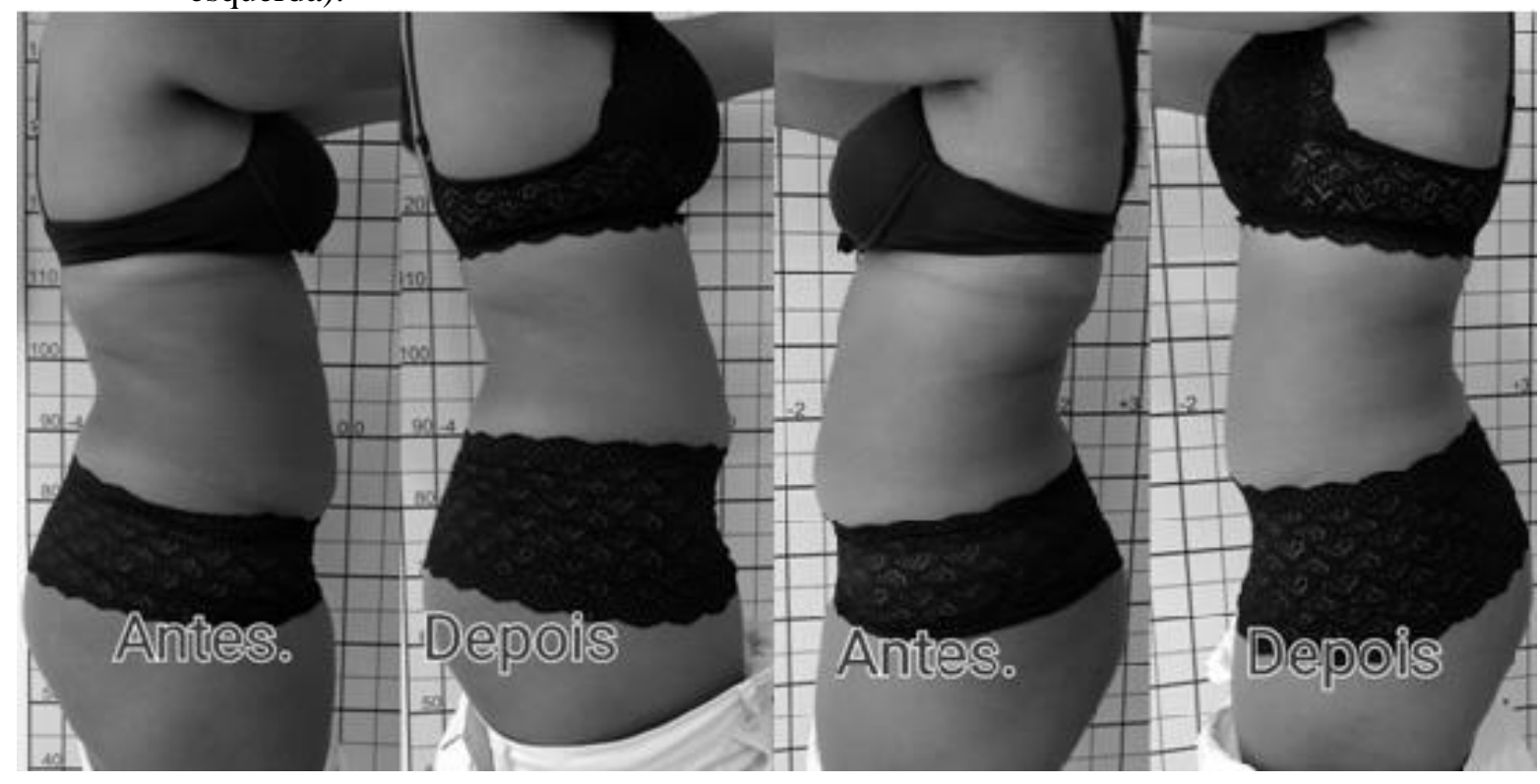




\section{Discussão}

Existem diversos aparelhos estéticos disponíveis para intensificar os resultados do tratamento da lipodistrofia localizada, porém, uma massagem modeladora bem feita associada a bons cosméticos também permitem um bom tratamento (OLIVEIRA, 2014).

A massagem modeladora é capaz de ajudar nos processos emagrecedores, uma vez que melhora a aparência da pele e seus contornos, estimula as funções viscerais, diminui a ansiedade e o estresse (TACANI, 2010). Borges (2006) a define como o uso de várias técnicas manuais que tem como finalidade a mobilização da gordura, aumento da circulação vascular periférica e eliminação de resíduos tóxicos. Tacani (2010) comenta que esse tipo de massagem usa manobras da massagem clássica estética com sentido centrípeto, ritmo constante, frequência de aproximadamente 5 segundos para cada movimento, intensidade e pressão moderadas.

É um procedimento que pode modelar o corpo em regiões nas quais há um acúmulo de gordura. Possui efeito termogênico, desintoxicante, enzimático, lipolítico, ortomolecular, reorganizador, vasodilatador e linfocinético; melhora a microcirculação e o retorno venoso; aumenta a produção de ATP em aproximadamente 500\%; aumenta o transporte de aminoácidos e da produção de proteínas com aumento da tonificação muscular; aumenta a circulação nos plexos vasculares com aumento da captação de oxigênio local; auxilia na normalização do metabolismo e promove regeneração celular. Tem ação prolongada (age por 48 horas) após sua aplicação, sendo que essa ação não é cumulativa (MENDES; COSTA, 2011).

Cassar (2001) explica que os benefícios da massagem na lipodistrofia localizada se justificam pela pressão mecânica aplicada que cria calor e hiperemia, ativa as células de gordura e consequentemente, provocando um maior gasto energético. Quando ocorre aumento na circulação dos vasos sanguíneos, a gordura tende a diminuir.

Borges (2006) observa que a massagem modeladora em conjunto com princípios ativos, como a centella asiática, cavalinha, cafeína e castanha da índia apresenta resultados mais significantes e satisfatórios.

A centella asiática possui ação antiedematosa e elastificante dos vasos sanguíneos, acelera a digestão das gorduras estimulando a ação das enzimas da pele (BIBIÁN, 2006). A cavalinha tem ação anti-radical livre e firmadora, pois fornece silício, que faz parte da estrutura do colágeno da derme (ADCOS, 2008). A cafeína é usada para potencializar a lipólise nos 
adipócitos, gerando a deterioração dos triglicerídeos, diminuindo assim o volume dos adipócitos (BIBIÁN, 2006). A castanha da índia tem ação antiedematosa, anti-inflamatória e venotônica (MARTINS; BRANDÃO, 2006).

Tanabe et al (2004) realizaram a mobilização mecânica de pré-adipócitos in vitro, o que diminuiu a diferenciação em adipócitos, servindo de base para a aplicação local desses estímulos na prevenção da lipodistrofia localizada.

Silva et al. (2009) aplicaram massagem modeladora em dez pacientes e concluiram que a técnica promove resultados satisfatórios na lipodistrofia localizada e é potencializada com a associação de ativos lipolíticos.

No estudo de Beneli et al. (1999), foi realizado 40 minutos de massagem mecânica no abdome e coxas de 10 pacientes obesas. Com base nos resultados obtidos, não houve alterações significativas de níveis plasmáticos que indicassem a ocorrência de lipólise, mas os autores relataram melhora dos contornos corporais e do aspecto do tecido adiposo subcutâneo.

Kim (2007) fez um estudo com 35 mulheres, nas quais aplicou massagem associada à aromaterapia uma vez por semana, durante seis semanas, no período pós-menopausa. O autor observou redução da circunferência da cintura, da gordura subcutânea abdominal e melhora da imagem corporal.

Fabris, Amorim e Watanabe (2009) selecionaram dez mulheres com idade entre 20 e 53 anos para realização da sua pesquisa. Nelas foi aplicado um creme redutor de gordura que continha cavalinha, cafeína, centella asiática e castanha da índia em sua formulação, na região abdominal por dez dias consecutivos. As perimetrias das voluntárias foram feitas antes de cada sessão do tratamento. Os autores observaram que o volume de gordura não diminuiu, porém, sete das dez mulheres mostraram resultados positivos quanto à perimetria.

Neves e Oliveira (2007) fizeram um estudo piloto com uma voluntária que foi submetida a dez sessões de ultrassom, massagem modeladora e cosméticos específicos. Foram feitas três perimetrias. A voluntária não perdeu peso, mas apresentou significante redução de medidas, diminuindo seis centímetros na primeira medida, dez centímetros na segunda e nove centímetros na terceira.

Em seu estudo, Tunay et al. (2010) fez uso da massagem modeladora em 60 mulheres com idade média de 40 a 43 anos, e relatou uma diminuição significante de $0,5 \mathrm{~cm}$ na perimetria 
de coxa e na espessura do tecido adiposo. Concluindo, assim, que a técnica de tratamento foi eficaz na diminuição do acúmulo de gordura.

Os resultados dos tratamentos estéticos para lipodistrofia localizada dependem principalmente do paciente. Este deve estar ciente que para ter um resultado potencializado, deve optar por bons hábitos alimentares e pela prática de atividades físicas (FABRIS; AMORIM; WATANABE, 2009).

\section{Conclusão}

Após a realização da pesquisa, conclui-se que a massagem modeladora é uma técnica eficaz no tratamento da lipodistrofia localizada e seu efeito é potencializado através da associação a princípios ativos lipolíticos, como cafeína, centella asiática, cavalinha e castanha da índia. Por meio da perimetria e do registro fotográfico, foi possível observar efeitos positivos no quadro de acúmulo de gordura que as voluntárias apresentavam na região abdominal. Com base nos resultados deste estudo, constatou-se que oito sessões foram suficientes para uma melhora satisfatória na redução de medidas.

\section{Referências}

ADCOS. Guia de produtos: reduxcel SML. Serra: Adcos, 2008.

BENELI L. et al. Endermologie: humoral repercussions and estrogen interaction. Aesthetic Plastic Surgery, v. 23, n. 5, p. 312-315, 1999.

BIBÍAN, R. P. Seis principios activos para el tratamiento de la celulitis. Sociedad Española de medicina estética. 2006. Disponível em: <HTTP:// www.seme.org/professionales/texto/txtCientifico.php?id=2> Acesso em 30 abr. 2017.

BORGES, F. S. Modalidades terapêuticas nas disfunções estéticas: dermato-funcional. São Paulo: Phorte, 2006.

CASSAR, M. Manual de Massagem Terapêutica. São Paulo: Manole, 2001. 
FABRIS, F. E.; AMORIM, P; WATANABE, E. Eficácia de um creme redutor de gordura e medidas na redução de perimetria abdominal: um estudo de caso. Acadêmica do curso de cosmetologia e estética da universidade do vale do itajai, UNIVALI, Balneário Camboriú, Santa Catarina, 2009.

FIGUEIREDO, S. G. D. O uso da cosmetologia associada à massagem modeladora no tratamento da celulite. Trabalho de conclusão de pós graduação de fisioterapia dermatofuncional - Faculdade Ávila. Manaus, 2011.

GOMES, R. K. DAMAZIO, M. G. Cosmetologia: Descomplicando os Princípios Ativos. 3. ed. São Paulo: Livraria Médica Paulista, 2009.

GUIRRO, E. C. O.; GUIRRO, R. R. J. Fisioterapia dermatofuncional: fundamentos, recursos e patologias. 3 ed. São Paulo: Manole, 2004, p. 49-380.

KIM, H. J. Effect of aromatherapy massage on abdominal fat and body image in postmenopausal women. Taehan Kanho Hakhoe Chi, v. 37, n. 4, p. 603-612, 2007.

MARTINS, E. L. P; BRANDÃO, M. G. L. Qualidade de amostras comerciais com aesculushippocastanum L. (Castanha da Índia). Revista Brasileira de Farmacognosia. Minas Gerais: Laboratório de Farmacognosia, Faculdade Farmacêutica, Universidade Federal de Minas Gerais, 2006.

MELLO, P. B. et al. Comparação dos efeitos da eletrolipólise transcutânea e prcutânea sobre a gordura localizada na região abdominal e de flancos através da perimetria e análise de bioimpedância elétrica. Fisioterapia Brasil, v. 11, n. 3, p. 198-203, maio-junho, 2010.

MENDES, T. C.; COSTA, E. G. Tratamento de fibro edema gelóide com o cosmético adenilciclase. Revista da Universidade Vale do Rio Verde, v. 1, n. 2, 2011.

NEVES, S. R.; OLIVEIRA, D. Eficácia da associação de técnicas manuais e eletrotermoterapia na redução de medidas do abdome. Revista Biologia Saúde Unisep, v. 1, n. 1, p, 67-71, 2008.

OLIVEIRA, A. L. De esteticista para esteticista: diversificando os protocolos faciais e corporais aplicados na área de estética. São Paulo: Matrix, 2014.

RIBEIRO, C. Cosmetologia aplicada a dermoestética. 1 ed. São Paulo: Pharmabooks, 2006.

SANDOVAL, A. E. P. Medicina do Esporte. Porto Alegre: Artmed, 2005.

SILVA, J, C. A. C. et al. Análise da eficácia da massagem modeladora na região abdominal de mulheres sadias. XIII Encontro Latino Americano de Iniciação Cientifica e IX Encontro Latino Americano de Pós-Graduação - Universidade do Vale do Paraíba, 2009. 
Id on Line Revista Multidisciplinar e de Psicoloqia

Id on Line Multidisciplinary and Psycology Journal

TACANI, P. M. et al. Efeito da massagem clássica estética em adiposidades localizadas: estudo piloto. Fisioterapia e Pesquisa, v. 17, n. 4, p. 352-357, 2010.

TANABE, Y. et al. Inhibition of adipocyte differentiation by mechanical stretching through ERK-mediated downregulation of PPARg2. Journal of Cell Science, v. 117, n. 16, p. 3605 3614, 2004.

TOGNI, A. B. Avaliação dos efeitos do ultrasom associado à fonoforese e endermologia no tratamento do fibro edema gelóide. Tubarão, SC, 2006. Monografia (Graduação). Curso de Fisioterapia, Universidade do Sul de Santa Catarina.

TUNAY, V. B. et al. Effects of mechanical massage, manual lymphatic drainage and connective tissue manipulation techniques on fat mass in women with cellulite. Journal of the European Academy of Dermatology and Venereology, v. 24, n. 2, p. 138-142, 2010.

Como citar este artigo (Formato ABNT):

MACHADO, Aiana Tátima O.M.; NOGUEIRA, Ana P. S.; LEÃO, Luciana T.de S.; SANTOS, Beluzia A.; PINHEIRO, Lívia M.G.; OLIVEIRA, Sirlândia S. Benefícios da Massagem Modeladora na Lipodistrofia Localizada. Id on Line Revista Multidisciplinar e de Psicologia, Maio de 2017, vol.11, n.35, p.542-553. ISSN: 1981-1179.

Recebido: 29.05.2017

Aceito: 31.05.2017 